\title{
Economic Dispatch Optimization Using Imperialist Competitive Algorithm (ICA) and compare with PSO algorithm result
}

\section{Saeid Jalilzadeh, Saman Nikkhah}

Associate Professor, Department of Electrical Engineering, University of Zanjan, Zanjan, Iran. jalilzadeh@znu.ac.ir

Research Scholar, Department of Electrical Engineering, University of Zanjan, Zanjan, Iran.

\section{ABSTRACT}

s.nikkhah@znu.ac.ir

\begin{abstract}
Measurement Imperialist Competitive Algorithm (ICA) is a population based stochastic optimization technique, originally developed by Eberhart and Kennedy, inspired by simulation of a social psychological metaphor instead of the survival of the fittest individual. In ICA, the system (imperialists) is initialized with a population of random solutions (colonies) and searches for optimal using cognitive and social factors by updating generations. ICA has been successfully applied to a wide range of applications, mainly in solving continuous nonlinear optimization problems. Based on the ICA, this paper discusses the use of ICA approach to optimize performance of economic dispatch problems. The proposed method is validated for a test system consisting of 13 thermal units whose incremental fuel cost function takes into account the valve-point loading effects.
\end{abstract}

\section{Indexing terms/Keywords}

Imperialist Competitive Algorithm (ICA), Particle Swarm Optimization (PSO), Economic Dispatch Problem (EDP).

\section{Council for Innovative Research}

Peer Review Research Publishing System

\section{Journal: INTERNATIONAL JOURNAL OF COMPUTERS \& TECHNOLOGY}

Vol. 15, No. 2

www.ijctonline.com, editorijctonline@gmail.com 


\section{INTRODUCTION}

The objective of the economic dispatch problem (EDP) of electric power generation, whose characteristics are complex and highly nonlinear, is to schedule the committed generating unit outputs so as to meet the required load demand at minimum operating cost while satisfying all unit and system equality and inequality constraints [1]. Improvements in scheduling the unit outputs can lead to significant cost savings. In traditional EDPs, the cost function of each generator is approximately represented by a simple quadratic function and is solved using mathematical programming based on several optimization techniques, such as dynamic programming, linear programming, homogenous linear programming, and nonlinear programming techniques [2]. However, none of these methods may be able to provide an optimal solution, for they usually get stuck at a local optimum.

Recently, as an alternative to the conventional mathematical approaches, modern heuristic optimization techniques such as simulated annealing, evolutionary algorithms, neural networks, and ant colony have been given much attention by many researchers due to their ability to find an almost global optimal solution in EDPs [3]-[6]. One of these modern heuristic optimization paradigms is the imperialist competitive algorithm optimization (ICA) [7]. ICA is a kind of evolutionary algorithm based on a population of colonies of any imperialists by the simulation of social behavior instead of the survival of the fittest individual. It is a population-based evolutionary algorithm. Similar to the other population-based evolutionary algorithms, ICA is initialized with colonies who related to own imperialist. Unlike the most of the evolutionary algorithms, each imperialist has own colonies and these colonies move toward their imperialist. Then imperialist competitive starts.

The approach of composite configuration by deterministic techniques combined with ICA algorithms is a promising alternative in optimization and must be evaluate. In this paper, an alternative hybrid method is proposed. The proposed technique is used in the learning phase (after the stopping criterion of ICA be satisfied) to solve the EDP associated with the valve-point effect. The difference increment is reduced as the optimization progresses, thereby avoiding some local minima, discontinuities, or nonsmooth regions that would trap a conventional gradient-based method.

An economic dispatch problem with 13 unit test system using nonsmooth fuel cost function [8] is employed in this paper for demonstrate the performance of the proposed hybrid method. The results obtained with the ICA approach were analyzed and compared with those obtained in recent literature. The rest of the paper is organized as follows: section II describes the EDP, while section III show the 13 thermal units information. Section IV presents the simulation results of the 13 thermal units whose incremental fuel cost function takes into account the valve-point loading effects. Lastly, section $\mathrm{V}$ outlines our conclusion.

\section{DESCRIPTION OF ECONOMIC DISPATCH PROBLEM}

Mathematically, The objective of the economic dispatch problem is to minimize the total fuel cost at thermal power plants subjected to the operating constraints of a power system. Therefore, it can be formulated mathematically with an objective function and two constraints. The equality and inequality constraints are represented by (1) and (2):

$$
\begin{aligned}
& \sum_{i=1}^{n} P_{i}-P_{L}-P_{D}=0 \\
& P_{i}^{\min } \leq P_{i} \leq P_{i}^{\max }
\end{aligned}
$$

In the power balance criterion, an equality constraint must be satisfied, as shown in (1). The generated power should be the same as the total load demand plus total line losses. The generating power of each generator should lie between maximum and minimum limits represented by (2), where $P_{i}$ is the power of generator (in MW) and $i$ is the number of generators in the system, $P_{D}$ is the system's total demand (in $\mathrm{MW}$ ), $P_{L}$ represents the total line losses (in MW) and $P_{i}^{\max }$ and $P_{i}^{\min }$ is the maximum and minimum power of the generating unit $i$ (in MW). The total fuel cost function is formulated as follows:

$\min f=\sum_{i=1}^{n} F_{i}\left(P_{i}\right)$

Where: $F_{i}$ is the total fuel cost for the generator unit $i$ (in $\$ / h$ ), which is defined by (4):

$$
F_{i}\left(P_{i}\right)=a_{i} P_{i}^{2}+b_{i} P_{i}+c_{i}
$$

where, $a_{i}, b_{i}$ and $c_{i}$ are cost coefficients of generator $i$. The generator costs are usually approximated using quadratic functions. However, it is more practical to consider the valve-point loading for fossil-fuel-based plants. In this context, a cost function is obtained based on the ripple curve for more accurate modeling. This curve contains higher order nonlinearity and discontinuity due to the valve point effect. One way of representing this effect is to model the generator cost curve by piecewise quadratic cost functions. A second approach is using a rectified sinusoidal function to represent the valve-point loading in the cost function [1]. In this case, (4) can be modified as:

$$
\tilde{F}_{i}\left(P_{i}\right)=F\left(P_{i}\right)+\left|e_{i} \sin \left(f_{i}\left(P_{i}^{\min }-P_{i}\right)\right)\right|
$$


or

$\tilde{F}_{i}\left(P_{i}\right)=a_{i} P_{i}^{2}+b_{i} P_{i}+c_{i}+\left|e_{i} \sin \left(f_{i}\left(P_{i}^{\min }-P_{i}\right)\right)\right|$

Where: $e_{i}$ and $f_{i}$ are coefficients of the valve point effect. Ignoring valve point effects, some inaccuracy would be introduced into the resulting dispatch. Hence, the total fuel cost that must be minimized, according to (3), is modified to:

$\min f=\sum_{i=1}^{n} \tilde{F}_{i}\left(P_{i}\right)$

Where $\tilde{F}_{i}$ is the cost function of generator $i$ (in $\$ / \mathrm{h}$ ) defined by (6). In the case study presented here, we disregarded the transmission losses.

\section{CASE STUDY OF EDP WITH 13 THERMAL UNITS}

This case study consisted of 13 thermal units of generation with the effects of valve point loading, as given in Table 1 . The data shown in Table 1 are also available in [8] and [15]. In this Table $a, b$ and $c$ are the cost coefficients of generator in (4). Also e and f are the coefficients of the valve points in (5) or (6). In this Table, the load demand expected to be determined was $p_{D}=1800 \mathrm{MW}$.

Table 1. Generating units data for the IEEE-39 bus test system

\begin{tabular}{cccccccc}
\hline $\begin{array}{c}\text { Thermal } \\
\text { Units }\end{array}$ & $p_{i}^{\min }$ & $p_{i}^{\max }$ & $a$ & $b$ & $c$ & $e$ & $f$ \\
\hline 1 & 0 & 680 & 0.00028 & 8.1 & 550 & 300 & 0.035 \\
2 & 0 & 360 & 0.00056 & 8.1 & 309 & 200 & 0.042 \\
3 & 0 & 360 & 0.00056 & 8.1 & 307 & 150 & 0.042 \\
4 & 60 & 180 & 0.000324 & 7.74 & 240 & 150 & 0.063 \\
5 & 60 & 180 & 0.000324 & 7.74 & 240 & 150 & 0.063 \\
6 & 60 & 180 & 0.000324 & 7.74 & 240 & 150 & 0.063 \\
7 & 60 & 180 & 0.000324 & 7.74 & 240 & 150 & 0.063 \\
8 & 60 & 180 & 0.000324 & 7.74 & 240 & 150 & 0.063 \\
9 & 60 & 180 & 0.000324 & 7.74 & 240 & 150 & 0.063 \\
10 & 40 & 120 & 0.00284 & 8.60 & 126 & 100 & 0.084 \\
11 & 40 & 120 & 0.00284 & 8.60 & 126 & 100 & 0.084 \\
12 & 55 & 120 & 0.00284 & 8.60 & 126 & 100 & 0.084 \\
13 & 55 & 120 & 0.00284 & 8.60 & 126 & 100 & 0.084 \\
\hline
\end{tabular}

\section{RESULTS}

The simulation results obtained from this paper are compared with other studies that given in References and showed in Table 2, which shows that the chaotic ICA succeeded in finding the best solution for the tested methods. However, the IF outperformed the other tested methods in terms of solution time. The best results obtained for solution vector $P_{i, i=1 . ., 13}$ with chaotic ICA with minimum cost of 17960.5358 \$/h is given in Table 3.

Table 2. Convergence results (50 runs) of a case study of 13 generating units With Valve Point

\begin{tabular}{ccccc}
\hline Optimization Method & Mean Time (s) & Minimum Cost (\$/h) & Mean Cost (\$/h) & Maximum Cost (\$/h) \\
\hline IF & 1.4 & 18812.3852 & 18962.0139 & 19111.6426 \\
PSO & 2.6 & 18874.7634 & 19159.3967 & 19640.4168 \\
Chaotic PSO & 3.3 & 18161.1013 & 18809.8275 & 19640.7556 \\
PSO-IF & 14.8 & 18605.1257 & 18854.1601 & 19111.6426 \\
Chaotic PSO-IF & 15.3 & 17963.9571 & 18725.2356 & 19057.2663 \\
ICA & ------ & 17960.5358 & 17963.3487 & 17967.9724 \\
\hline
\end{tabular}


Table 3. Active power of each generator with $p_{D}=1800 \mathrm{MW}$

\begin{tabular}{cccc}
\hline Active power & Generation (MW) & Power & Generation (MW) \\
\hline $\mathrm{P}_{1}$ & 628.3173125 & $\mathrm{P}_{8}$ & 60 \\
$\mathrm{P}_{2}$ & 149.5996451 & $\mathrm{P}_{8}$ & 109.8500877 \\
$\mathrm{P}_{3}$ & 222.8040779 & $\mathrm{P}_{10}$ & 40 \\
$\mathrm{P}_{4}$ & 109.8415858 & $\mathrm{P}_{11}$ & 40 \\
$\mathrm{P}_{5}$ & 109.8580679 & $\mathrm{P}_{12}$ & 55 \\
$\mathrm{P}_{6}$ & 109.8662358 & $\mathrm{P}_{13}$ & 55 \\
$\mathrm{P}_{7}$ & 109.8629873 & $\sum_{i=1}^{13} p_{i}$ & 1800 \\
& & & \\
\hline
\end{tabular}

Table 4. Comparison of case study results for fuel costs presented in the literature

\begin{tabular}{|cc|}
\hline Optimization Technique & Case Study with 13 Thermal Units \\
\hline Evolutionary programming [10] & 17994.07 \\
\hline Particle swarm optimization [1] & 18030.72 \\
\hline Hybrid evolutionary programming with SQP [1] & 17991.03 \\
\hline Hybrid particle swarm with SQP [1] & 17969.93 \\
\hline Hybrid Chaotic Particle Swarm Optimizer & 17963.9571 \\
\hline Best result of this paper using ICA approach & $\mathbf{1 7 9 6 0 . 5 3 5 8}$ \\
\hline
\end{tabular}

Table 4, compares the results obtained in this paper with those of other studies reported in the literature. Note that in studied case, the result reported here using chaotic ICA is comparatively lower than recent studies presented in the literature.

\section{CONCLUSION}

The contribution of this paper is the using of ICA to solve an EDP. This method was applied to optimize for fuel cost of 13 thermal units. Results were analyzed and compared with other heuristic optimization algorithm. The results show that the proposed method has better influence than other heuristic optimization to solve an EDP. Another profit of the proposed method was the high accuracy and speed of that in reaching the optimal value.

\section{REFRENCES}

[1] A. Pereira-Neto, C. Unsihuay and O. R. Saavedra, "Efficient evolutionary strategy optimization procedure to solve the nonconvex economic dispatch problem with generator constraints," IEE Proceedings-Generation, Transmission, and Distribution, vol. 152, no. 5, pp. 653-660, 2005.

[2] L. S. Coelho and V. C. Mariani, "Combining of chaotic differential evolution and quadratic programming for economic dispatch optimization with valve-point effect," IEEE Transactions on Power Systems, vol. 21, no. 2, pp. 989-996, 2006.

[3] T. Yalcinoz and H. Altun, "Power economic dispatch using a hybrid genetic algorithm," IEEE Power Engineering Review, March, pp. 59-60, 2001. 
[4] Y. H. Song and C. S. V. Chou, "Large-scale economic dispatch by artificial ant colony search algorithms," Electric Machines and Power Systems, vol. 27, pp. 679-690, 1999.

[5] M. Basu, "A simulated annealing-based goal-attainment method for economic emission load dispatch of fixed head hydrothermal power systems," International Journal of Electrical Power \& Energy Systems, vol. 27, no. 2, pp. 147153, 2005.

[6] Balakrishnan, P. S. Kannan, C. Aravindan and P. Subathra, "On-line emission and economic load dispatch using adaptive Hopfield neural network," Applied Soft Computing, vol. 2, no. 4, pp. 297-305, 2003.

[7] E. Atashpaz_gargari, c. Lucas, Imprrialist Competitive Algorithm: an algorithm for optimization inspired by imperialistic competition, (ICA) IEEE CEC 2007.

[8] N. Sinha, R. Chakrabarti, and P. K. Chattopadhyay, "Evolutionary programming techniques for economic load dispatch," IEEE Transactions on Evolutionary Computation, vol. 7, no. 1, pp. 83-94.

[9] D. Stoneking, G. Bilbro, R. Trew, P. Gilmore, and C. T. Kelley, "Yield optimization using a GaAs process simulator coupled to a physical device model," Proceedings IEEE/Cornell Conference on Advanced Concepts in High Speed Devices and Circuits, Ithaca, NY, USA, pp. 374-383, 1991.

[10] D. B. Bertsekas, "On the Goldstein-Levitin-Polyak gradient projection method," IEEE Transactions on Automatic Control, vol. 21, no. 2, pp. 74-184, 1976.

[11] P. Gilmore and C. T. Kelley, "An implicit filtering algorithm for optimization of functions with many local minima," SIAM Journal of Optimization, vol. 5, no. 2, pp. 269-275, 1995.

[12] P. Gilmore and C. T. Kelley, "An implicit filtering algorithm for optimization of functions with many local minima," Technical Report CRSC-TR94-23, North Carolina State University, Center for Research in Scientific Computation, USA, 1994.

[13] K. P. Wong and Y. W. Wong, "Genetic and genetic/simulated-annealing approaches to economic dispatch," IEE Proc. Control, Generation, Transmission and Distribution, vol. 141, no. 5, pp. 507-513, 1994.

[14] T. D. Choi, O. J. Eslinger, C. T. Kelley, J. W. David, and M. Etheridge, "Optimization of automotive valve train components with implicit filtering," Optimization and Engineering, vol. 1, no. 1, pp. 9-27, 2000.

[15] Leandro dos Santos Coelho and Viviana Cocco Mariani, "Economic Dispatch Optimization Using Hybrid Chaotic Particle Swarm Optimizer,"1-4244-0991-8/07/\$25.00/C2007 IEEE.

\section{Author' biography with Photo}

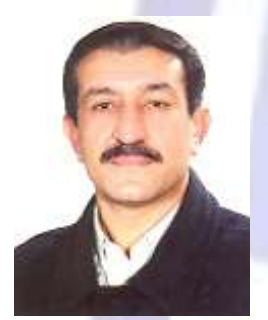

Saeid Jalilzadeh received the B.Sc. and M.Sc. degree in electrical engineering from

University of Tabriz, Tabriz, Iran, in 1987 and 1991, and Ph.D. degrees in electrical engineering from Iran University of Science and Technology, Tehran, Iran, in 2005.

$\mathrm{He}$ is currently a Professor of electrical engineering at University of Zanjan. His research interests are in power system operation and planning and electric machines.

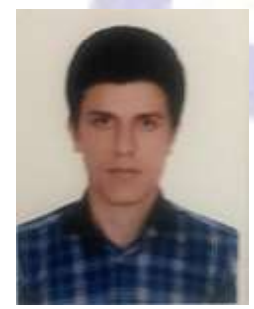

Saman Nikkhah received the B.Sc. degree in electrical engineering from

University of Urmia, Urmia, Iran, in 2013. He is currently pursuing the M.Sc. degree at University of Zanjan, Zanjan, Iran. His research interests are in power system operation and planning. 